\title{
Spiral Growth and Step Edge Barriers
}

\author{
Alex Redinger, ${ }^{1,2}$ Oliver Ricken, ${ }^{1}$ Philipp Kuhn, ${ }^{3}$ Andreas Rätz, ${ }^{4}$ Axel Voigt, ${ }^{4}$ Joachim Krug, ${ }^{3}$ and Thomas Michely ${ }^{1}$ \\ ${ }^{1}$ II. Physikalisches Institut, Universität zu Köln, 50937 Köln, Zülpicher Strasse 77, Germany \\ ${ }^{2}$ I. Physikalisches Institut, RWTH Aachen, 52056 Aachen, Germany \\ ${ }^{3}$ Institut für Theoretische Physik, Universität zu Köln, 50937 Köln, Zülpicher Strasse 77, Germany \\ ${ }^{4}$ Institut für Wissenschaftliches Rechnen, Technische Universität Dresden, Zellescher Weg 12-14, 01062 Dresden, Germany
}

(Received 6 September 2007; revised manuscript received 14 November 2007; published 24 January 2008)

The growth of spiral mounds containing a screw dislocation is compared to the growth of wedding cakes by two-dimensional nucleation. Using phase field simulations and homoepitaxial growth experiments on the $\operatorname{Pt}(111)$ surface we show that both structures attain the same large scale shape when a significant step-edge barrier suppresses interlayer transport. The higher vertical growth rate of the spiral mounds on $\mathrm{Pt}(111)$ reflects the different incorporation mechanisms for atoms in the top region and can be formally represented by an enhanced apparent step-edge barrier.

When a screw dislocation intersects a crystal surface, a step emerges from the intersection point which cannot be removed by growth or evaporation. Under deposition the step turns around the intersection point, resulting in a characteristic spiral mound. This mechanism was conjectured by Frank in 1949 to account for the enormous discrepancy between experimentally observed crystal growth rates and the predictions of two-dimensional nucleation theory by allowing for growth even at very low supersaturations, where nucleation is extremely unlikely $[1,2]$. Since then, spiral mounds have been recognized as a ubiquitous feature of many growth systems ranging from high temperature superconductors [3] to biominerals [4] and organic thin films $[5,6]$.

Theoretically, predicting the shape of a growth spiral involves a moving boundary value problem for the adatom concentration in a complex geometry [7], which has been treated by approximate analytical calculations $[8,9]$ as well as by numerical phase field techniques [10]. These approaches result in a simple conical mound shape with a constant step spacing, which is proportional to the step radius of curvature near the spiral core and decreases as a power law with increasing deposition flux.

In the growth of organic thin films frequently spiral mounds are observed whose shapes are inconsistent with this classical scenario. They possess small flat plateaus at the top, a slope (or step spacing) that varies nonmonotonically with the distance from the spiral core, and they are typically separated by deep grooves $[5,6]$. These shapes are strikingly reminiscent of the mound structures, often referred to as wedding cakes, that form during growth on dislocation-free metal surfaces [11-14] when the mass transport between different layers is suppressed by a strong step-edge barrier (SEB). It is then plausible to hypothesize that the unconventional spiral mounds observed on organic thin films may be related to a SEB, which has been proposed to exist on the basis of various pieces of evidence $[5,15-18]$.
In the present work we therefore investigate experimentally and theoretically the hitherto disregarded situation of spiral growth in the presence of a SEB. Our starting point is the observation that an atom finding itself on the hillside of a spiral mound, far away from the core, diffuses in an environment that is indistinguishable from that on the hillside of a wedding cake consisting of concentric circular islands [8]. The overall shape of the spiral mound must therefore reflect the strength of the SEB in the same way as for a wedding cake.

This is illustrated in Fig. 1 for a growth spiral generated numerically using a phase field model $[19,20]$. The model is a diffuse interface approximation of the moving boundary value problem first formulated by Burton, Cabrera, and Frank [2]. Key parameters are the surface diffusion coefficient $D$ and the deposition flux $F$, as well as the kinetic coefficients $k_{ \pm}$governing the attachment of atoms to a step from the lower $\left(k_{+}\right)$and upper $\left(k_{-}\right)$terrace, respectively. Step thermodynamics enters through the step stiffness $\gamma$ and the equilibrium adatom concentration $\rho^{*}$. In the phase field approximation the crystal surface is represented by a continuous field $\phi(\vec{r}, t)$ which couples to the adatom concentration and is subjected to a multiwell potential that pins $\phi$ to the preferred integer values representing the discrete surface heights. The SEB is incorporated through
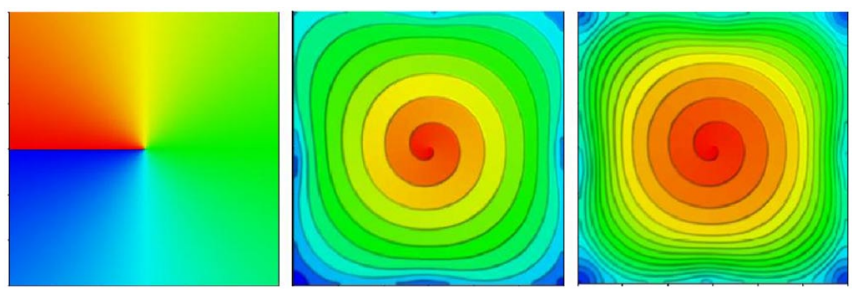

FIG. 1 (color online). Growth spirals obtained from phase field simulations with $D=10, F=0.2, \gamma=1, \rho^{*}=0.1, k_{+}=10$, $k_{-}=1$. Panels show the surface before growth (left) and after deposition of $140 \mathrm{ML}$ (middle) and $300 \mathrm{ML}$ (right), respectively. 
a mobility function that is minimal at the steps and asymmetric with respect to half-integer values of $\phi$. In the numerical implementation the model is nondimensionalized by scaling lengths and times with suitable microscopic quantities.

A screw dislocation is introduced by the shift $\phi \rightarrow \phi-$ $\theta(\vec{r}) / 2 \pi$, where $\theta(\vec{r})$ is the planar rotation angle around the dislocation core $[10,20]$. In the absence of a SEB the simulation of the model converges to a conical mound with a spatially constant, time-independent slope. By contrast, the spiral mound displayed in Fig. 1 has a flattop and curved hillsides which steepen indefinitely with increasing deposition flux, up to the point where further steepening is limited by the intrinsic step width of the phase field. This is precisely the behavior expected from the analogy with wedding cakes $[11,14]$.

Despite the similarity in the large scale shape, it is clear that the growth mechanism near the top of the structure is completely different for spiral mounds and for wedding cakes. Even the answer to the simple question as to which of the two grows faster under a given set of conditions is not obvious. The growth of wedding cakes is governed by the rate of two-dimensional nucleation of islands on the top terrace, which is enhanced by the confinement of atoms due to the SEB [21]. In contrast, atoms landing near the top of the spiral mound can avoid the SEB by moving around the core, which should reduce the local adatom concentration and hence the growth rate. On the other hand, the indelible presence of the step emanating from the screw dislocation obviates the need for two-dimensional nucleation, which should increase the rate of vertical growth. When the adatom concentration in our simulations was allowed to become sufficiently large so that islands nucleated deterministically, spiral mounds were generally seen to grow higher than regular wedding cakes [20], indicating that the second effect dominates.

To experimentally compare the two growth mechanisms under identical conditions, we have devised a setup in which they are simultaneously realized on the Pt(111) surface. The experiments were performed in an ultrahigh vacuum variable temperature scanning tunneling microscopy apparatus with a base pressure in the $10^{-11} \mathrm{mbar}$ range [22]. Cleaning of the $\mathrm{Pt}(111)$ sample was accomplished by cycles of flash annealing to $1273 \mathrm{~K}$ and sputtering by a mass separated $5 \mathrm{keV} \mathrm{Ar}^{+}$ion beam. In order to fabricate a surface with screw dislocation lines intersecting the surface in a suitable areal density, prior to growth the procedure outlined in [23] was applied. Helium was implanted at room temperature into the sample by a $4.5 \mathrm{keV}$ $\mathrm{He}^{+}$ion beam incident at an angle of $76^{\circ}$ with respect to the surface normal and with a dose of $3.8 \times 10^{20}$ ions $/ \mathrm{m}^{2}$. Subsequently the surface was annealed for $120 \mathrm{~s}$ to $T=$ $800 \mathrm{~K}$. The growth of the precipitating He bubbles gives rise to dislocation loop punching. Dislocation reactions and glide towards the surface result partially in loop an- nealing (adatom island formation) but partially also in separated pairs of screw dislocation intersection points with the surface (step dipoles). Onto this surface Pt was evaporated with a typical rate of $1 \times 10^{-2} \mathrm{ML} / \mathrm{s}$, where 1 monolayer (ML) denotes the surface atomic density of $\mathrm{Pt}(111)$. The deposited amounts $\Theta$ range from 1 to $100 \mathrm{ML}$ and temperatures $T$ from 250 to $500 \mathrm{~K}$. After deposition the morphology was quenched to avoid changes due to surface diffusion.

Figure 2(a) shows the morphology after deposition of a total coverage $\Theta$ of $39 \mathrm{ML}$ at $400 \mathrm{~K}$. As in previous experiments on dislocation-free surfaces [13,14], a large number of mounds are formed that exhibit flattops and are separated by deep grooves. Two of the mounds visible in Fig. 2(a) are significantly higher than all others; their heights exceed the surrounding mounds by 4-6 ML. Closer inspection reveals that both carry a screw dislocation intersection point in their center, as exemplified by the inset. Figure 2(b) displays the mound profiles obtained from the height distributions of the spiral mound labeled
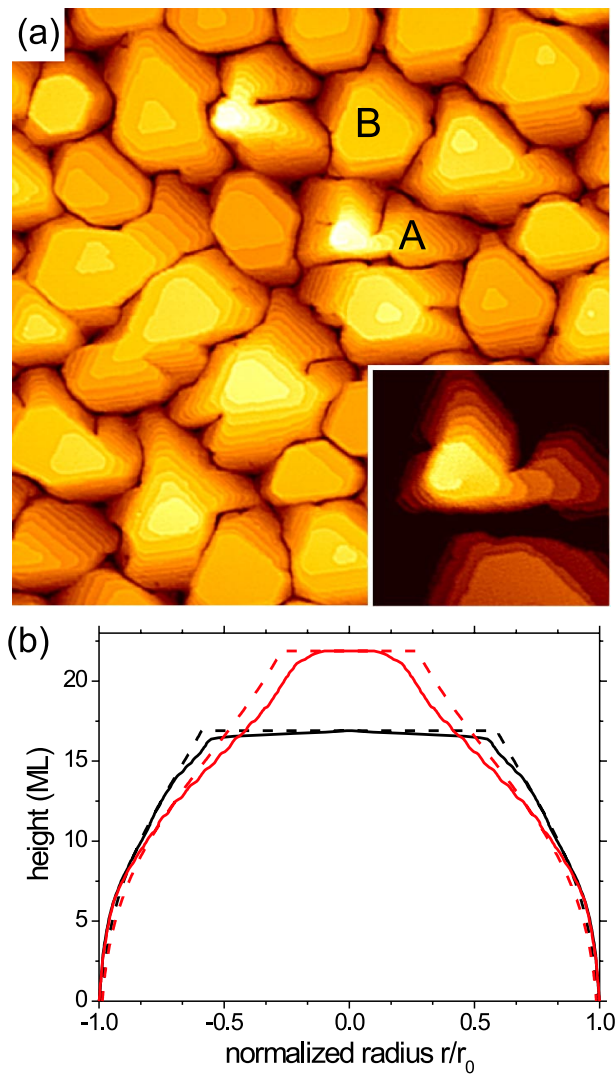

FIG. 2 (color online). (a) Morphology of Pt(111) after deposition of $39 \mathrm{ML}$ at $400 \mathrm{~K}$ on a substrate area with screw dislocation intersection points. Image size $1750 \AA \times 1750 \AA$. Inset: enlarged view of the top area of the spiral mound labeled $A$. (b) Radially averaged height distributions of the spiral mound labeled $A$ in (a) (full red/gray line) and the wedding cake labeled $B$ in (a) (full black line). The fits to the shape profiles according to the analytic model are plotted as dashed lines. 
$A$ and the wedding cake labeled $B$ in Fig. 2(a). The profiles are obtained by redistributing the layer coverages into a stack of circular islands. Both mounds clearly show flattop plateaus. The spiral mound profile displays in addition a change from positive curvature close to the top to negative curvature close to the edge. Such an inflection point is characteristic for mounds grown in the presence of a large SEB [14].

The dashed lines in Fig. 2(b) represent fits to the function $\theta(h)=1-C\{1+\operatorname{erf}[(h-\Theta) / \sqrt{\Theta}]\}$, where $\theta(h)$ is the fractional coverage at height $h$ and erf denotes the error function. The width of the mound at height $h$ is proportional to $\sqrt{\theta(h)}$. This equation follows from a simple model for the growth of wedding cakes [24], in which mass transport between all layers except the top one is completely suppressed and a new top terrace nucleates when the current one has reached a critical coverage $\theta_{c}$ [14]. The only fit parameter $C$ is related to $\theta_{c}$ and is most simply determined from the total height of the mound. While the agreement with the experimental data is not perfect, the fit is satisfactory in view of the crudeness of the model and the large number of factors that have been neglected in its application. For example, repulsive step-step interactions come into play when the steps on the hillside approach each other and tend to reduce the mound slope, as observed in Fig. 2(b).

Via the theory of second layer nucleation [14,21], the critical coverage $\theta_{c}$ can be linked to the additional SEB $\Delta E_{S}$, which is defined as the difference between the activation energy for a descending hop into the step position compared to a hop within the layer [25]. A large value of $\Delta E_{S}$ enhances the confinement of adatoms on the top terrace, which increases the probability for nucleation, decreases $\theta_{c}$, and increases the height of the mound. Using the known data for adatom diffusion on $\mathrm{Pt}(111)$ [14], the fits shown in Fig. 2(b) translate into the estimates $\Delta E_{S}=0.22 \mathrm{eV}$ for the wedding cake (mound $B$ ) and $\Delta E_{S}=0.34 \mathrm{eV}$ for the spiral mound (mound $A$ ). Although spiral mounds obviously do not grow by twodimensional nucleation, we can formally represent the experimentally observed faster growth of the spiral mounds by an increase of the apparent SEB by $0.12 \mathrm{eV}$.

Another prediction of the model is that the height difference between two mounds characterized by different values of $C$ or $\theta_{c}$ grows proportional to $\sqrt{\Theta}$. This prediction should also apply to the average height difference $\Delta h$ between spiral mounds and wedding cakes. Figure 3 shows experimental data for $\Delta h$ obtained by measuring the extra height of a spiral mound with respect to the heights of the wedding cakes being its direct neighbors. This quantity was averaged for each temperature $T$ and coverage $\Theta$ over an ensemble of spiral mounds. Indeed Fig. 3 shows that $\Delta h$ grows roughly proportional to $\sqrt{\Theta}$ for the two temperatures analyzed.

As a further test of the concept of an increase in the apparent SEB for spiral mounds, we have determined the

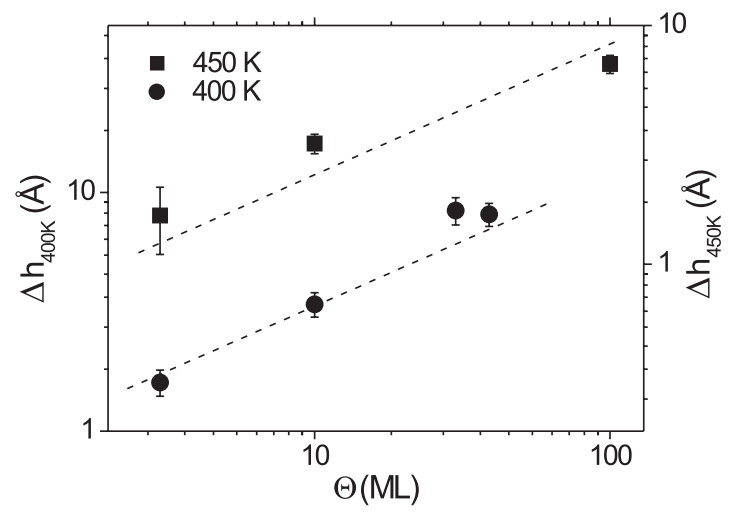

FIG. 3. Average height difference $\Delta h$ between spiral mounds and wedding cakes for $400 \mathrm{~K}(\boldsymbol{O})$ and $450 \mathrm{~K}(\boldsymbol{\square})$ as a function of $\Theta$. The dashed lines correspond to $\Delta h \propto \sqrt{\Theta}$ as predicted by the mound growth model.

sizes of the flattop region for mounds grown at various temperatures with a total coverage of $10 \mathrm{ML}$ [see Figs. 4(a)-4(c)]. To characterize the extension of the top area for spiral mounds, the sum of the lengths of step segments 7 to 12 of the spiral is used as sketched in Figs. 4(d) and 4(e). This corresponds to the perimeter of the base terrace, the terrace below the top terrace of a wedding cake, which is known to show less scatter than the top terrace size itself [21]. Using formulas of second layer nucleation theory [14], the base terrace perimeter translates
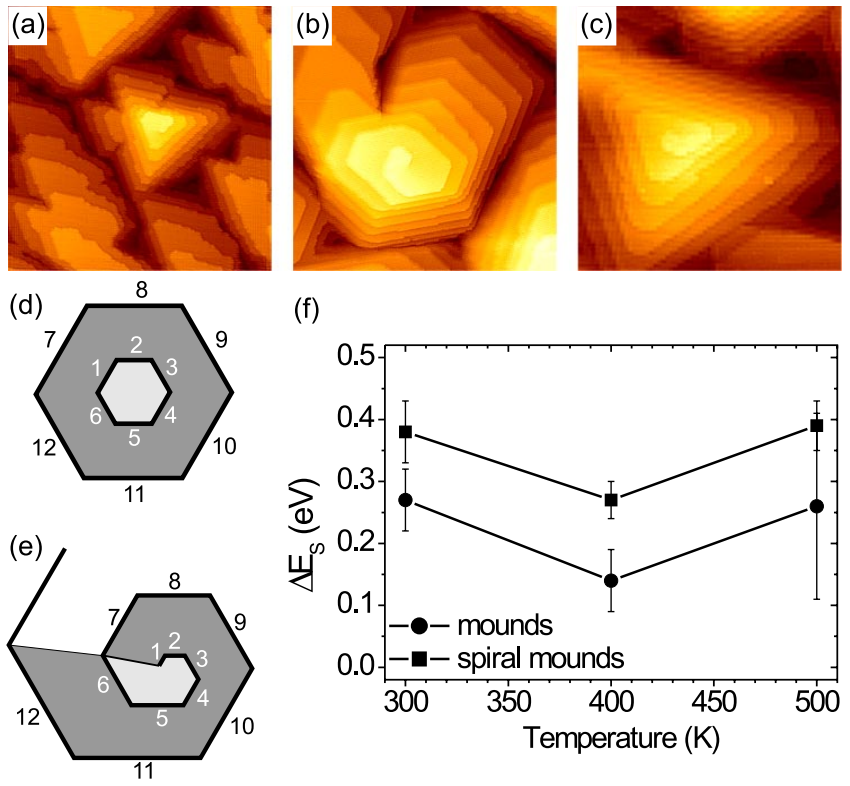

(f)

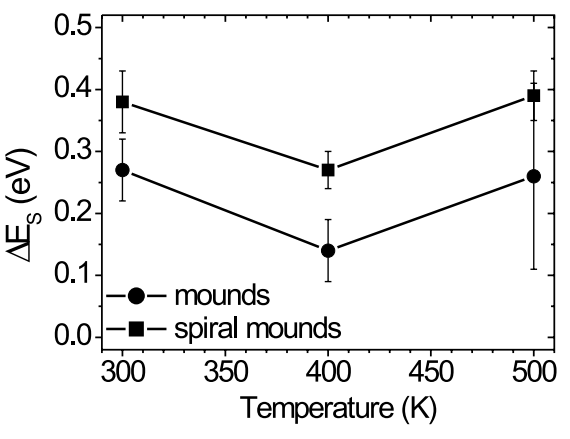

FIG. 4 (color online). Examples of spiral mounds after deposition of $10 \mathrm{ML}$ at (a) $300 \mathrm{~K}$, (b) $400 \mathrm{~K}$, (c) $500 \mathrm{~K}$. Image size is always $400 \AA \times 400 \AA$. Schematic top view sketch of the top area of (d) a wedding cake and (e) a spiral mound. The numbers label the step segments. (f) Results for $\Delta E_{S}$ for wedding cakes (O) for the three growth temperatures investigated together with the apparent $\Delta E_{S}$ for spiral mounds ( $\square$ ) grown simultaneously. 
into estimates for $\Delta E_{S}$ for the two kinds of mounds, which are displayed in Fig. 4(f). We find a consistent increase of the apparent SEB of spiral mounds by $0.11-0.13 \mathrm{eV}$, in good agreement with the result obtained from the mound shapes in Fig. 2.

The results presented so far raise an obvious question: is the faster growth of spiral mounds observed on $\mathrm{Pt}(111)$ a generic feature, or is it specific to this growth system? To provide at least a qualitative answer, note first that only the fate of the adatoms arriving in the top region [the areas shaded light gray in Figs. 4(d) and 4(e)] determines the shapes and heights of mounds, given that $\Delta E_{S}$ is significant, because then adatoms arriving on the slopes of a mound are incorporated with a probability near unity into the ascending step bounding their terrace. In contrast, adatoms arriving near the top of a mound are able to reach a lower layer, either because the top terrace on which they land is small (in the case of a wedding cake) or because they can move around the spiral core. Thus the area of the top region is directly proportional to the effective downhill flux of adatoms. The smaller the top region, the higher the mound and the closer the mound shape is to the statistical growth limit, in which the layer coverages have a Poisson distribution [11,14].

Understanding the difference between the two kinds of mounds therefore amounts to understanding the physics that determines the size of the top area. For wedding cakes the key process is two-dimensional nucleation [14,21]. For the spiral mounds on $\mathrm{Pt}(111)$, the appropriate physical picture is that of a polygonized spiral growing by the motion of straight segments that follow the crystallographic directions of the surface [7]. The scale of the top region is then set by the length $l_{c}$ that the stationary segment 1 in Fig. 4(e) has to reach before it can grow by incorporating adatoms, thus taking over the role of segment 2. Hence $l_{c}$ is the critical segment length for onedimensional nucleation, and it is determined by the atomistic processes of step-edge diffusion and corner rounding [14]. Applying the kinematic approach of [7] to the fcc(111) surface, one finds that $l_{c}$ equals the length of segment 2, which does not change in time. In the present case this yields the estimate $l_{c} \approx 23 \pm 6 \AA$, which is much smaller than the average top terrace size of a wedding cake. This discussion makes it clear that for a system with a different hierarchy of diffusion processes the effect of a spiral step might be qualitatively different. If the activation energy for step-edge diffusion is small and the SEB is large, $l_{c}$ could be large compared to the size of a top terrace at second layer nucleation, and wedding cakes could be higher than spiral mounds. In fact, this has to be the case in the limit $\Delta E_{S} \rightarrow \infty$, where the top terrace of a wedding cake shrinks to a point [11] while the spiral mounds maintain a finite top plateau of size $\sim l_{c}$.
In summary, screw dislocations and step-edge barriers are two of the most important factors shaping the morphology of growing crystals. Here we have explored their cooperative effect, thus clarifying the significance of the anomalous spiral mounds observed in organic films and providing a basis for the controlled use of kinetic growth instabilities for the patterning of thin film surfaces.

We acknowledge useful discussions with C. Busse, M. Beigmohamadi, A. Farahzadi, P. Niyamakom, E.D. Williams, M. Wuttig, and W. Kalb. A. Redinger acknowledges support from RWTH Aachen.

[1] W. K. Burton, N. Carbrera, and F.C. Frank, Nature (London) 163, 398 (1949).

[2] W. K. Burton, N. Carbrera, and F. C. Frank, Phil. Trans. R. Soc. A 243, 299 (1951).

[3] C. Gerber et al., Nature (London) 350, 279 (1991).

[4] H. H. Teng et al., Science 282, 724 (1998).

[5] R. Ruiz et al., Chem. Mater. 16, 4497 (2004).

[6] M. Beigmohamadi et al., Phys. Status Solidi RRL 2, 1 (2008).

[7] I. V. Markov, Crystal Growth for Beginners (World Scientific, Singapore, 2004), 2nd ed., pp. 218.

[8] T. Surek, J. P. Hirth, and G. M. Pound, J. Cryst. Growth 18, 20 (1973).

[9] J. P. van der Eerden, J. Cryst. Growth 53, 305 (1981).

[10] A. Karma and M. Plapp, Phys. Rev. Lett. 81, 4444 (1998).

[11] J. Krug, J. Stat. Phys. 87, 505 (1997).

[12] P. Politi, J. Phys. I 7, 797 (1997).

[13] M. Kalff et al., Surf. Sci. 426, L447 (1999).

[14] T. Michely and J. Krug, Islands, Mounds and Atoms Springer Series in Surface Science Vol. 42 (Springer, New York, 2004).

[15] B. Krause et al., Europhys. Lett. 65, 372 (2004).

[16] S. Zorba, Y. Shapir, and Y. Gao, Phys. Rev. B 74, 245410 (2006).

[17] A. C. Mayer et al., Phys. Rev. B 73, 205307 (2006).

[18] M. Fendrich and J. Krug, Phys. Rev. B 76, 121302 (2007).

[19] F. Otto et al., Nonlinearity 17, 477 (2004).

[20] A. Rätz, Ph.D. dissertation, University of Bonn, 2007.

[21] J. Krug, P. Politi, and T. Michely, Phys. Rev. B 61, 14037 (2000).

[22] M. Bott, T. Michely, and G. Comsa, Rev. Sci. Instrum. 66, 4135 (1995).

[23] T. Michely and G. Comsa, J. Vac. Sci. Technol. B 9, 862 (1991).

[24] The equation applies in the limit of large $\Theta$, where the fact that the height distribution of a wedding cake is discrete, in contrast to the continuous distribution of a spiral mound, is irrelevant.

[25] We use $\Delta E_{S}$ as effective barrier averaging over the atomistic details of the step configuration, which in fact give rise to a variation of the barrier with step atomic structure and also neglect details of the potential energy landscape at the step edge (compare [14]). 